\title{
Nanostructured Sensors for Determination of 3-(3,4-Dichlorophenyl)-1,1- Dimethylurea Based in Molecularly Imprinted Polymers (MIPs) Deposited in Screen Printed Carbon Nanotubes
}

\author{
R. A. Fernández-Cori ${ }^{\mathrm{a}}$, J. C. Morales-Gomero ${ }^{\mathrm{a}, \mathrm{b}}$, B. C. Huayhuas-Chipana ${ }^{\mathrm{a}}$, M. D. P. \\ Taboada-Sotomayor ${ }^{\mathrm{c}}$, J. G. Ruíz-Montoya ${ }^{\mathrm{a}}$ \\ ${ }^{\text {a }}$ Facultad de Ciencias, Universidad Nacional de Ingeniería, Rímac, Lima, Peru \\ ${ }^{\mathrm{b}}$ Facultad de Ingeniería Industrial, Universidad de Lima, Santiago de Surco, Lima, Peru \\ ${ }^{c}$ Department of Analytical Chemistry, Institute of Chemistry, UNESP - Universidade \\ Estadual Paulista, São Paulo, 14801-970 Araraquara, Brazil
}

\begin{abstract}
A sensor based on molecularly imprinted polymers (MIP) was developed for determination of 3-(3,4-Dichlorophenyl)-1,1dimethylurea (diuron). The MIP were synthesized by free radical polymerization in wich diuron acted as template, methacrylic acid (MAA) acted as functional monomer and ethylene glycol dimethacrylate (EGDMA) acted as crosslinker. For construction of the sensor, glassy carbon electrode was modified with Nafion ${ }^{\circledR}$ and MIP/NIP (non imprinted polymer) to see the contribution of MIP, as a film, in the diuron recognition. Differential Pulse Voltammetry was used to the quantification of diuron in a range of response $0.4-1.2 \mathrm{~V}$ in a $\mathrm{PBS}(\mathrm{pH}=8)$. All parameters affecting the sensor were optimized. The sensor showed a linear response from $1.00 \times 10^{-5}$ to $9.616 \times 10^{-5} \mathrm{M}$ in PBS $0.1 \mathrm{M}(\mathrm{pH}=8)$. The limit of detection was $0.58 \times 10^{-5} \mathrm{~mol} \mathrm{~L} \mathrm{~L}^{-1}$. The nanostructured sensor was prepared using carbon nanotubes and showed a 4 times greater response than the glassy carbon, showing promising results.
\end{abstract}

\section{Introduction}

Diuron (3-(3,4-Dichlorophenyl)-1,1-Dimethylurea: DU) is a phenylurea herbicide which inhibits photosynthesis by preventing oxygen production (1) and blocks the electron transfer at the level of photosystem II of photosynthetic microorganisms and plants. This compound has been used to control a wide variety of annual and perennial broadleaf and grassy weeds, as well as mosses (2). Although its use on crops can improve yields and profit margins, diuron (figure 1) can cause environmental impacts, affecting algae, fungi, plants, and mammals. In humans, exposure to diuron results in the formation of methemoglobin in the blood, as well as liver and spleen abnormalities. Diuron also acts as an endocrine disruptor that interferes in the processes of release, transport, and disposal of natural hormones in body (2-4).

Diuron has low solubility in water, and when applied to the soil tends to accumulate. The half-life of the herbicide ranges from 90 to 180 days in soil (5), so its high persistence also that in heavy rainfall causes its leaching cause the groundwater pollution 
due to its slow rate of removal; it can therefore be found in many environments, including soil, sediments, and water (2).<smiles>CN(C)C(=O)Nc1ccc(Cl)c(Cl)c1</smiles>

Figure 1. Chemical structure of diuron

Due to the serious effects that pesticides such as diuron can have on humans and other living organisms, is necessary the monitoring of levels of these substances in the environment. A variety of analytical methods for the monitoring of diuron have been reported in the literature, these include chromatographic, spectrometric, fluorometric, capillary electrophoretic, and electrochemical techniques (5).

In recent decades scientists have taken interest in electrochemical sensor based on molecularly imprinted polymers which could offer good limits of detection, at low costs, with the possibility of easy miniaturization and automation (6). Also, sensors using acrylic or vinyl MIPs are reported to have good stability during prolonged storage (more than 6 months in many cases), as expected for a highly cross-linked polymer. This type of transduction is especially attractive with a view to making readily available a range of small devices based on recognition by a templating effect in relevant applications, such as biomarkers in clinical chemistry, environmental control in the field, on-line quality control in the pharmaceutical industry or detection of food fraud (6-9). In addition, the electrodes modified with single walled nanotubes were widely used to develop new type of biosensors with highly improved sensitivity and performance $(10,11)$.

The objective of this experiment was to develop a nanostructured sensor based on molecularly imprinted polymer (MIP) for determination of diuron. The molecularly imprinted polymer was previously synthesized ant then integrated on glassy carbon electrode to observer the binding capacity of MIP like a film. Subsequently, screen printed single walled carbon nanotubes electrodes were modified to observe the contribution of its high electrical properties in response of nanostructured sensor.

\section{Experimental}

\section{$\underline{\text { Reagents and materials }}$}

Diuron, Nafion ${ }^{\circledR}$, methacrylic acid (MAA), ethylene glycol dimethacrylate (EGDMA), azobisisobutyronitrile (AIBN), $\mathrm{KH}_{2} \mathrm{PO}_{4}$ and $\mathrm{K}_{2} \mathrm{HPO}_{4}$ were acquired from Sigma-Aldrich. Chloroform, methanol, acetic acid, acetone and acetonitrile were obtained from Merck. A $2.02 \times 10^{-3} \mathrm{~mol} \mathrm{~L}^{-1}$ diuron stock solution was prepared by dissolving $0.0047 \mathrm{~g}$ of the compound in $10.0 \mathrm{~mL}$ of acetonitrile and kept in the dark at $4^{\circ} \mathrm{C}$. Phosphate buffer solution ( $\mathrm{PBS}, 50 \mathrm{mM}, \mathrm{pH} 8.0$ ) was prepared with $\mathrm{KH}_{2} \mathrm{PO}_{4}$ and $\mathrm{K}_{2} \mathrm{HPO}_{4}$. All aqueous solutions were prepared with ultrapure water $(>18 \mathrm{M} \Omega \mathrm{cm})$ obtained from a Milli-Q Plus (Millipore) purification system. 
$\underline{\text { Instruments and measurements }}$

Scanning electron microscope (SEM) images were acquired with an XL30 ESEM FEG field emission scanning electron microscope. All Fourier transform infrared (FTIR) spectroscopic measurements were performed on a Bruker Vertex 70 spectrometer. Ultraviolet visible (UV-vis) absorption spectra were recorded by a U-3900 Hitachi spectrometer. Cyclic voltammetry (CV), differential pulse voltammetry (DPV) were performed using a Dropsens $\mu$ Stat 400 and were carried out with a typical three-electrode system, with a platinum wire as auxiliary electrode and an $\mathrm{Ag} \mid \mathrm{AgCl}(1 \mathrm{M} \mathrm{KCl})$ reference electrode, the working electrodes were bare and modified screen printed single-walled carbon nanotube electrode ( $\mathrm{SWCNTE}, \mathrm{d}=4 \mathrm{~mm}$ ) which was obtained from DropSens and glassy carbon electrode ( $\mathrm{GCE}, \mathrm{d}=4 \mathrm{~mm})$.

\section{$\underline{\text { Synthesis of MIP and NIP }}$}

The schematic diagram for the preparation of MIPs is shown in Fig. 2. 0.1mmol of diuron and $0.5 \mathrm{mmol}$ of MAA were dissolved in $5 \mathrm{~mL}$ of chloroform. The mixture was shaken in a water bath at $25{ }^{\circ} \mathrm{C}$ for $4 \mathrm{~h}$. Subsequently, $2.0 \mathrm{mmol}$ of EGDMA and $0.03 \mathrm{mmol}$ of AIBN were added into the system and the mixture was sonicated, degassed with nitrogen for $5 \mathrm{~min}$, and placed in a water bath at $60^{\circ} \mathrm{C}$ for $24 \mathrm{~h}$. The obtained products (MIPs contained the template) were dried at room temperature for $24 \mathrm{~h}$, grinded and sieved. For comparison, non-molecular imprinted polymers (NIPs) were prepared under the same conditions without the template (DU).

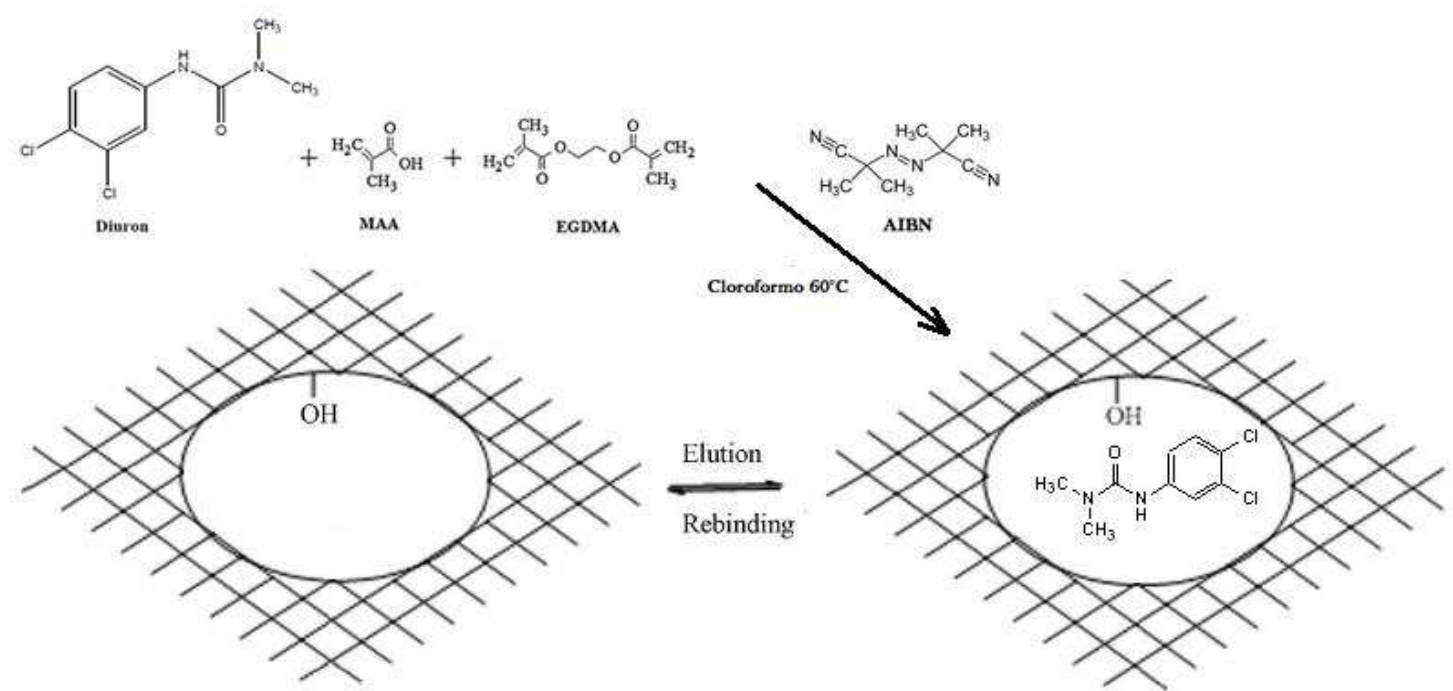

Figure 2. Schematic illustration of synthesis of molecularly imprinted polymer

\section{Electrochemical measurements}

A bare glassy carbon electrode was first polished using $0.05 \mu \mathrm{m}$ alumina slurry, following by thoroughly flushing with ultrapure water, and then cleaned ultrasonically in acetone and ultrapure water successively. Screen printed single-walled carbon nanotube electrode was cleaned using cyclic voltammetry by potential scanning repeatedly between 
0.4 and $1.2 \mathrm{~V}$ by 20 times. MIPs $(4 \mathrm{mg})$ were dispersed in $0.4 \mathrm{~mL}$ of methanol by ultrasonication for $20 \mathrm{~min}$. The above suspension $(20 \mu \mathrm{L})$ was mixed with $20 \mu \mathrm{L}$ of Nafion ${ }^{\circledR}$ and $10 \mu \mathrm{L}$ of this mixture was dropped onto the electrode surface and dried at room temperature overnight. Removal of the template molecules was achieved by cyclic voltammetry to glassy carbon electrode modified with MIP (GCE-MIP) and screen printed carbon nanotube electrode modified with MIP (SWCNTE-MIP) in $50 \mathrm{mM}$ PBS. The cyclic voltammetry was carried out by potential scanning repeatedly between 0.4 and $1.2 \mathrm{~V}$ until there was no signal of DU. After extraction of the templates, the electrodes were rinsed thoroughly with ultrapure water and then submitted to binding and selective recognition experiments. The modified electrodes were incubated in $10 \mathrm{~mL}$ of different concentrations of DU solution (recognition experiments) for 3 min and examined by DPV.

\section{Results and discussion}

The morphology of MIP and NIP unleached was examined by SEM. As shown in Fig. $3 \mathrm{a}$ and $\mathrm{b}$, the MIP and NIP exhibited irregular shape in morphology conformed by aggregate of sheets which had several hundreds of nanometers in size, both MIP and NIP had the same morphology indicating that diuron doesn't affect the morphology of polymer.
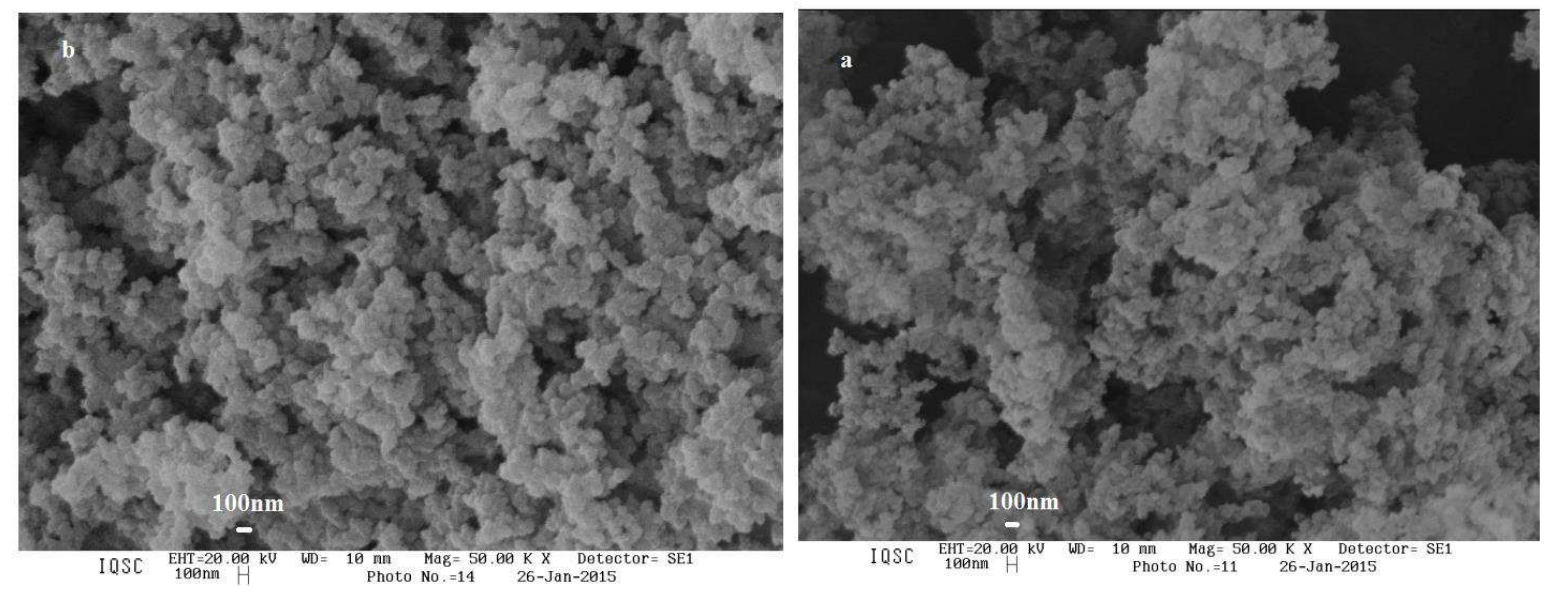

Figure 3. SEM images of MIP (a) and NIP (b)

The molecular structures of MIP and NIP were characterized by FTIR spectroscopy. The content of the remaining carbon-carbon double bonds in the MIP is an important indication of polymerization extent. No band is present in the region of $1648-1638 \mathrm{~cm}^{-1}$, indicating the absence of vinyl groups in the polymerized materials (12). As shown in Fig. 4, the band at $2975 \mathrm{~cm}^{-1}$ and peak at $1457 \mathrm{~cm}^{-1}$ could be assigned to the stretching and scissor vibration of $\mathrm{C}-\mathrm{H}$ of methylene groups respectively, the peak at $1720 \mathrm{~cm}^{-1}$ is attributed to the stretching vibration of $\mathrm{C}=\mathrm{O}$ bonds in carbonyl groups and the peak at $1389 \mathrm{~cm}^{-1}$ is attributed to the symmetrical deformation of $\mathrm{C}-\mathrm{H}$ bonds of methyl groups (13). The results clearly indicated that both MIP and NIP has molecular structure similarity indicating that diuron doesn't affect the polymeric structure. 


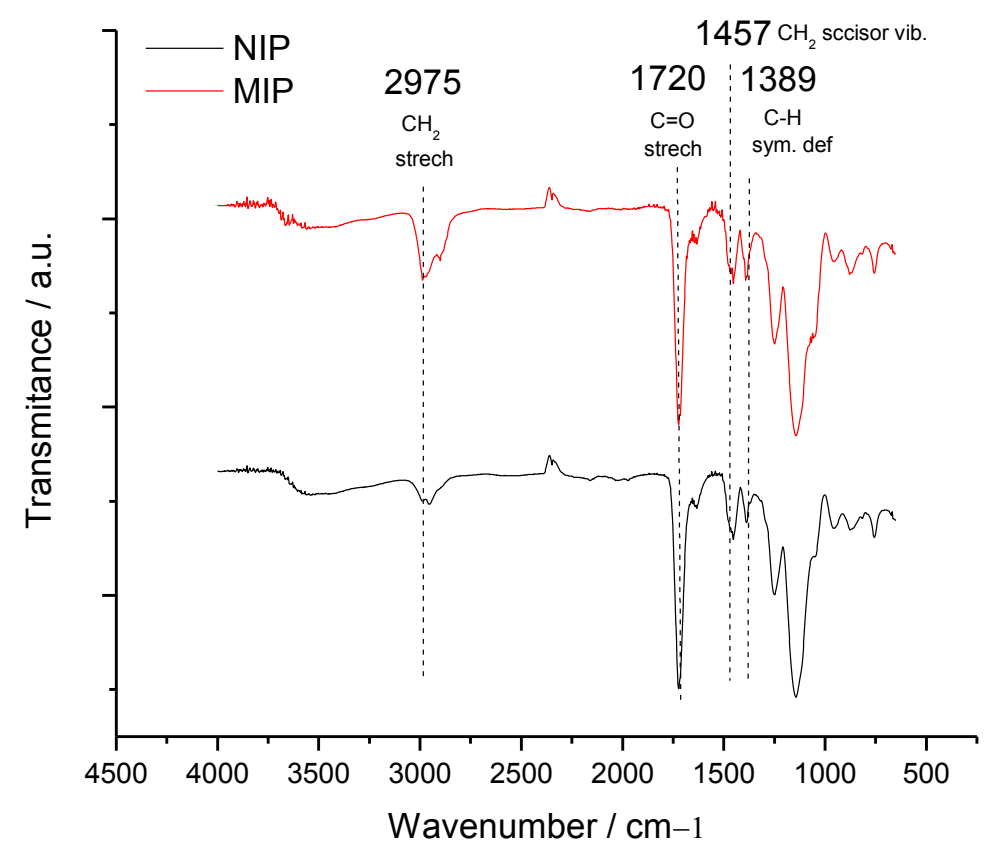

Figure 4. FTIR spectra of MIP with diuron (red line) and NIP (black line)

Both FTIR and SEM analysis demonstrated that presence of diuron didn't affect the polymeric structured, so to demonstrate the presence of diuron in MIP, part of MIP was washed with methanol/acetic acid (4:1 v/v) and the washout solution was analyzed by UV/Vis spectrometry, according to Fig. $5 \mathrm{~b}$, a peak at wavelength $250 \mathrm{~nm}$ corresponding to absorbance of diuron present in the washout solutions (14), its indicate the presence of diuron in the polymeric matrix.

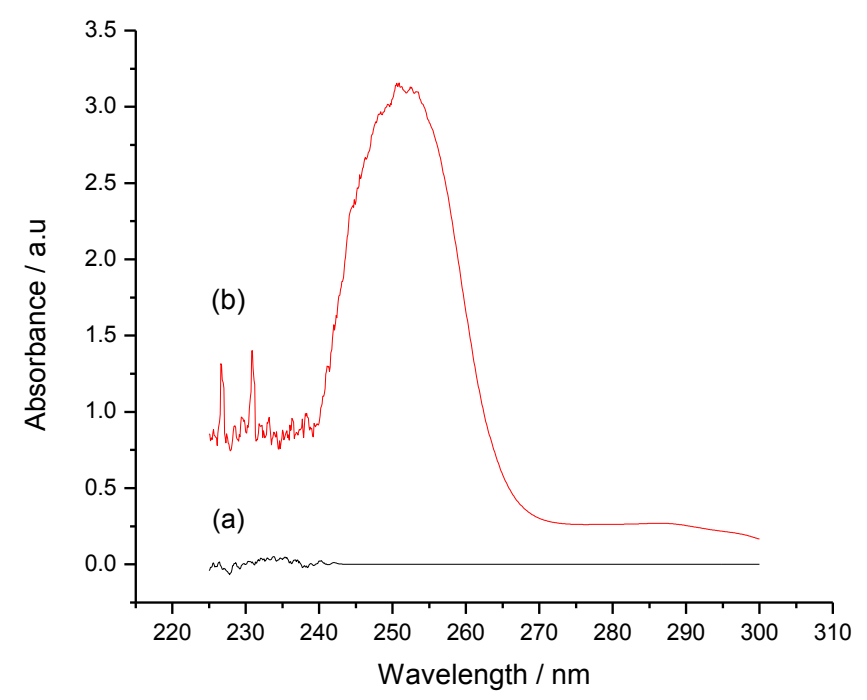

Figure 5. UV/Vis spectrum of methanol/acetic acid $(4: 1 \mathrm{v} / \mathrm{v}$, a) and solution after the washing of MIP (b) 


\section{$\underline{\text { Template extraction and adsorption of MIP }}$}

The conventional method for template extraction is using organic reagents or buffer solution as eluent. However, it is time consuming and the template cannot be removed entirely. Since the oxidation of diuron was chemically irreversible under the experimental conditions (15), DU molecules could easily leach out the binding sites during the electrochemical reaction. In this work, voltammetry cyclic was performed to extract DU molecules from the imprinted polymers as is observed in Fig. 6a, in the decrease a peak oxidation at $1.09 \mathrm{~V}$ belonging to irreversible oxidation of DU, thus DU could be purged rapidly and completely. For comparison, cyclic voltammetry was applied to GCE-NIP (Fig. 6b) which didn't show any oxidation peak, also the electrochemical measurements were carried out in PBS free of DU, and this implied that the oxidation peaks were entirely due the DU embedded inside the imprinted polymers film. As a consequence, these results demonstrated that the binding affinity of the imprinted film was due to the specific sites formed by the imprinting effect.
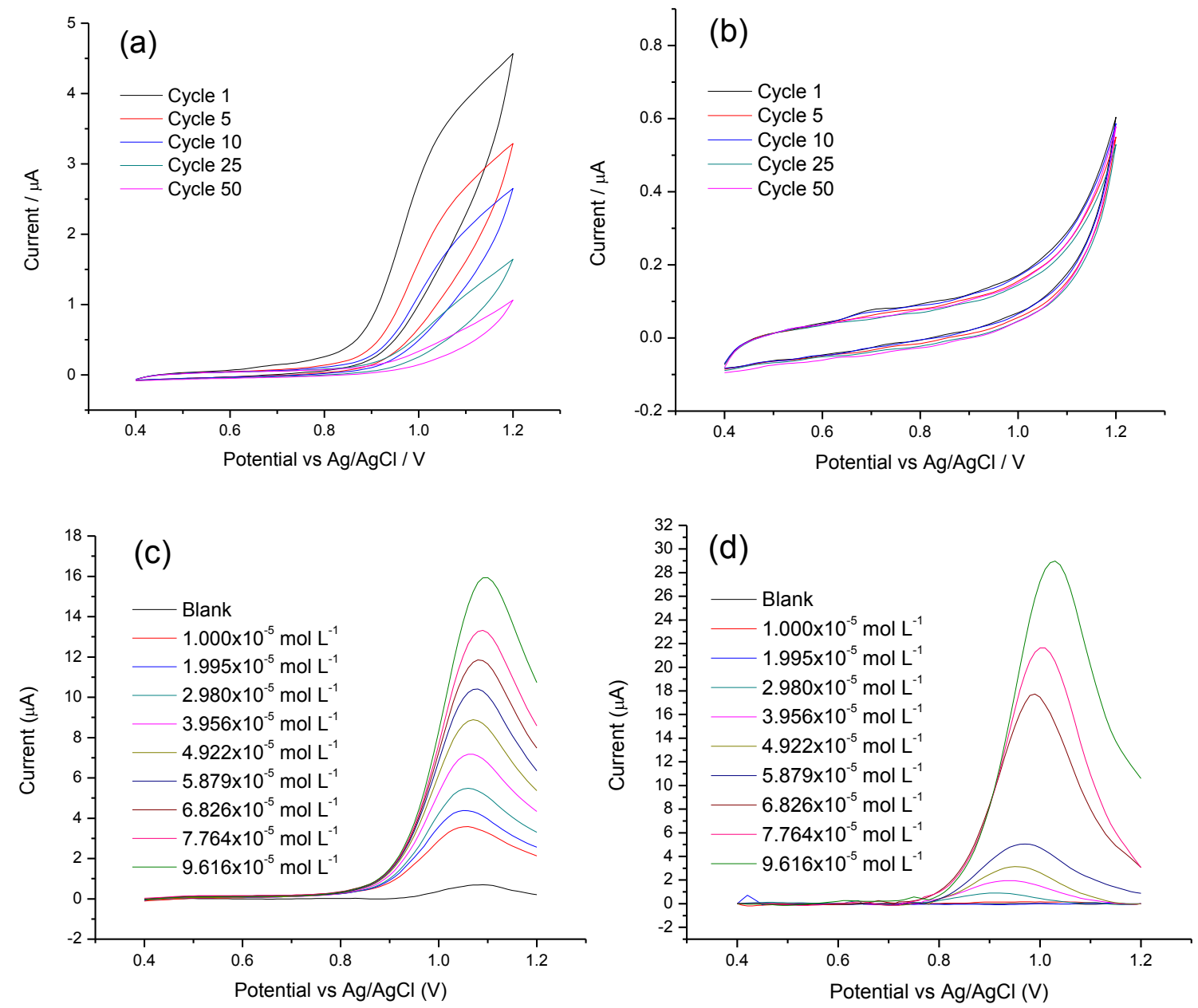


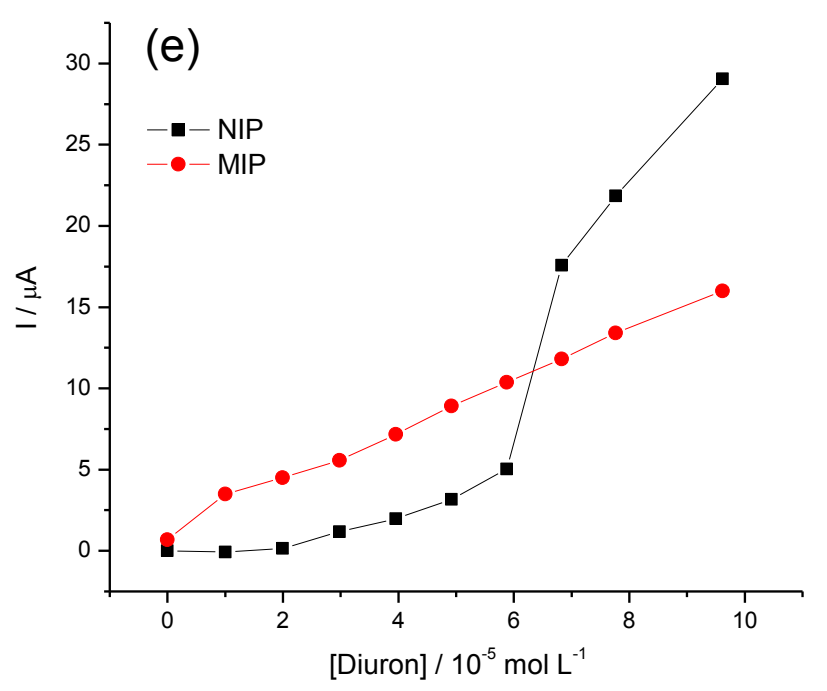

Figure 6. Cyclic voltammetry of GCE-MIP (a) and GCE-NIP (b) in PBS $50 \mathrm{mM}$ pH 8.0.

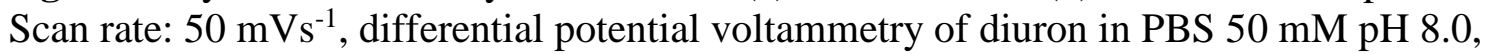
with concentration varying from 0 to $9.616 \times 10^{-5} \mathrm{~mol} \mathrm{~L}^{-1}$ using GCE-MIP (c) and GCENIP (d). Scan increment: $10 \mathrm{mV}$, pulse amplitude: $100 \mathrm{mV}$, Scan rate: $50 \mathrm{mVs}^{-1}$, and the comparison (e) between GCE-MIP (red line) and GCE-NIP (black)

In the figure $6 \mathrm{c}$, we obtained as limit of detection $0.58 \times 10^{-5} \mathrm{~mol} \mathrm{~L}-1$, the sensibility of the sensor was $1.5505 \mathrm{uA} / 10^{-5} \mathrm{~mol} \mathrm{~L}-1$. In addition, the higher oxidation current of diuron in GCE-MIP indicated that the binding capacity was better than NIP (Fig.6d,e) in the concentration range from 1.000 to $5.879 \times 10^{-5} \mathrm{~mol} \mathrm{~L}^{-1}$, this confirm that GCE-MIP has a specific binding capacity for the template molecule. Also the oxidation current of diuron in GCE-MIP follow a linear tendency in this concentration range, which could obey the Nernst-Planck equation indicating the diuron diffusion to the surface electrode (16) compared with GCE-NIP which not follow a linear tendency, also to higher concentrations than $5.879 \times 10^{-5} \mathrm{~mol} \mathrm{~L}^{-1}$ the oxidation current of diuron in GCE-NIP is higher than GCE-MIP, it is possibly because to lower concentrations the film of NIP is impeding the free diffusion of diuron to surface electrode and diuron molecules is being retained in the film of NIP so that at high concentrations, all the diuron molecules retained diffuse to the surface electrode causing a sharp increase in the oxidation current and a linear tendency a high concentrations indicating a free diffusion of diuron.

\section{$\underline{\text { Nanostructured sensor modified with MIP }}$}

Taking advantage of the high electrical properties of single walled carbon nanotube, a screen printed single walled carbon nanotube electrode was modified with MIP (SWCNTE-MIP) and NIP (SWCNTE-NIP) and cyclic voltammetry in PBS $50 \mathrm{mM}$ $\mathrm{pH} 8.0$ containing $6.826 \times 10^{-5} \mathrm{~mol} \mathrm{~L}^{-1}$ (Fig. 7) was performed to observe if the nanostructured electrode help to improve the electrochemical response $y$ resolve the problem that happened with the binding capacity of GCE-MIP to concentrations higher than $5.879 \times 10^{-5} \mathrm{~mol} \mathrm{~L}^{-1}$. According to Fig. 7 appear an oxidation peak at $0.808 \mathrm{~V}$ which is lower compared to GCE-MIP $(1.09 \mathrm{~V})$, this indicate that nanostructured electrode has catalytic properties, also according to table I the oxidation current of diuron in SWCNTE- 
MIP is as four times higher than response of SWCNTE-NIP, this attributed a its high electrical properties (10).

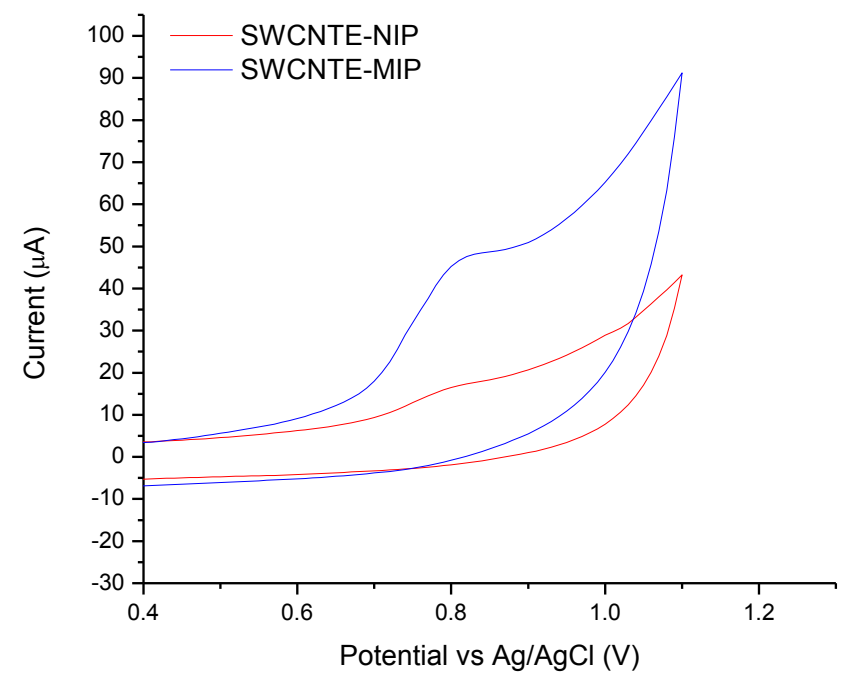

Figure 7. Voltammograms of molecularly imprinted polymer (blue line) and nonmolecularly imprinted polymer (red line) in a solution of diuron containing $6.826 \times 10^{-5}$ $\mathrm{mol} \mathrm{L}^{-1}$ in PBS 0.1 M, pH 8.

TABLE I. Current oxidation of diuron in nanostructured electrode modified with MIP and NIP

\begin{tabular}{cc}
\hline Electrode & Current oxidation of diuron $(\boldsymbol{\mu A})$ \\
\hline SWCNTE-MIP & 32.7 \\
SWCNTE-NIP & 7.72 \\
\hline
\end{tabular}

\section{Conclusion}

A molecularly imprinted polymer selective recognition of DU was successfully synthesized via free radical polymerization method. Previously analysis using glassy carbon electrode indicate that MIP like a film can recognize DU, and determine DU with a linear range from 1.000 to $5.879 \times 10-5 \mathrm{~mol} \mathrm{~L}^{-1}$. Screen printed singles walled carbon nanotubes electrode was modified with MIP and NIP demonstrating the great support of high electrical properties of nanostructured electrode in the determination of diuron to higher concentrations, also in the decrease of oxidation potential of diuron indicating that has catalytic properties so the imprinted film building on a nanostructured should be promising as it provides more sensibility for template recognition and better potential for diuron determination. This strategy can be further expected to be used to fabricate various molecular imprinting-based on nanostructured sensors for advanced applications. Furthermore, the molecular imprinting techniques discussed herein could also find applications in the fields of separation, trace detection, and environmental monitoring. 


\section{Acknowledgments}

The authors are most grateful to Facultad de Ciencias of Universidad Nacional de Ingeniería, Department of Analytical Chemistry of Institute of Chemistry of UNESP Universidade Estadual Paulista, and FINCyT Project Contract 228 IA-2013 for financial support.

\section{References}

1. J. S. C. Wessels and R. Van der Veen, Biochem. Biophys. Acta, 19, 548 (1956).

2. S. Giacomazzi and N. Cochet, Chemosphere, 56, 1022 (2004).

3. T. Prichard, J. Troiano, J. Marade, F. Guo, M. Canevari, J. Environ. Qual., 34, 2005-2017 (2005).

4. G. S. A. Ferdandes, A. C. Arena, C. D. B. Fernandez, A. Mercadante, L. F. Barbisan, W. G. Kempinas, Reprod. Toxicol, 23, 106 - 112 (2007).

5. A. Wong, M. R de Vasconcelos-Lanza, M. D. P. Taboada-Sotomayor, J. Electroanal. Chem., 690, 83-88 (2013).

6. M. C. Blanco-López, M. J. Lobo-Castañón, A. J. Miranda-Ordieres and P. TuñónBlanco, Trends Anal. Chem., 23, 1, 36-48 (2004).

7. T. A. Sergeyeva, S. A. Piletsky, A. A. Brovko, E. A. Slichenko, L. M. Sergeeva and A. V. El'skaya, Anal. Chim. Acta, 392, 105 (1999).

8. T. Panasyuk-Delaney, V. M. Mirsky, M. Ulbritch and O. S. Wolfbeis, Anal. Chim. Acta, 435, 157 (2001).

9. T. Panasyuk-Delaney, V. M. Mirsky, O. S. Wolfbeis, Electroanalysis, 14, 221 (2003).

10. P. R. Bandaru, J. Nanosci. Nanotechnol., 7, 2-4 (2007).

11. H. Zhanga, G. Liua and C. Chai, Sensors and Actuators B, 168, 103 (2012).

12. J. R. Santos, P. R. Lima, C. R. T. Tarleyb and L. T. Kubota, J. Braz. Chem. Soc, 20, 5, 820-825 (2009).

13. R. M. Silverstein, F. X. Webster and D. J. Kiemle, Spectrometric indentification of organic compounds, p.121-124, John Wiley \& Sons, Inc., USA (2005).

14. A. H. Kamell and F. M. Al-Romian, Int. J. Chem. Mater. Sci., 1, 1, 1-12 (2013).

15. G. Byzynski-Soares, W. Tadeu-Lopes da Silva and C. M. P. Vaz, Sensors Letters, 9, 1-8 (2011).

16. A. J. Bard and L. R. Faulkner, Electrochemical Methods: Fundamentals and Applications, p. 29, John Wiley \& Sons, Inc., USA (2001). 